\title{
Analysis the Structure of SAM and Cracking Password Base on Windows Operating System
}

\author{
Jiang Du and Jiwei Li
}

\begin{abstract}
Cracking Windows account password is critical to the forensic analyst. The general methods of decipher password include clean the password, guess by social engineering, mathematical analyzing, exhaustive attacking, dictionary attacking and rainbow tables algorithm. This paper provides the details about the Security Account Manager(SAM) database and describes how to get the user information from SAM and cracks account password of Windows 10 that the latest operating system of Microsoft.
\end{abstract}

Index Terms-SAM, decipher password, crack password, Windows 10.

\section{INTRODUCTION}

In the process of computer forensic the analyst need to enter the Windows operating system by cracking windows account password to collect evidence at times. Within the family of Windows operating systems, like mostly used Windows 7, Windows 8 and the latest Windows 10, the Security Account Manager(SAM) database was used to store user's login information and passwords which encrypted by NT-hash [1]. This paper analysis the structure of the SAM that come from Windows 10 and makes an experiment to obtain the user's account information from the SAM and crack the account password. During the research and experimentation stage, the following software will be utilized:

1) VM ware Workstation 9.0.0 build- 812388 was used to create a virtual machine;

2) Mount image pro v5.0.6 was used to mount E01 disk image file as a physical disk;

3) Red Hat Enterprise Linux Server release 7.0 was created by VMware Workstation to run the programs;

4) Access Data FTK imager 3.0.0.1443 was used to create an E01 disk image file from Microsoft Windows 10 operating system;

5) The SAM file is come from the Windows 10 which mounted by Mount image pro v5.0.6;

\section{WINDOWS REGISTRY OVERVIEW}

The Windows registry is a central hierarchical database used in the entire operating system of Microsoft to store users' information, applications and hardware devices [2], like the mostly used in Windows XP, Windows 7, Windows 8 and the

Manuscript received January 4, 2016; revised April 12, 2016.

Jiang Du and Jiwei $\mathrm{Li}$ are with College of Computer Science and Technology, Chongqing University of Posts and Telecommunications, Chongqing 400065, China (e-mail: clouddu@163.com, cloudleejw@163.com). latest Windows 10, the registry contains information that Windows continually references during operation, such as profiles for each user, the applications installed on the computer and the types of documents, property sheet settings of folders and application icons, what hardware exists on the system, and the ports that are being used [3]. On disk, the Windows registry is not only a large file but a set of discrete files called hives. Each hive is a hierarchical tree which identified by a root key to provide access to all sub-keys in the tree up to 512 levels deep.

\section{SEcurity AcCount MAnAger (SAM)}

Security Account Manager (SAM) is a database used to store user account information, including password, account groups, access rights, and special privileges in Windows operating system. In the registry, all of the users include the general users and the administrators can not read the SAM file except the users who gain the system privileges. So if the general user and administrator want to read the SAM file they have to gain read access by changing the access control list of those registry keys with the permission of the administrative privileges. And the file can be found in \%SystemRoot/system32/config/SAM\% and it mounted on HKLM/SAM and HKLM/SE-CURITY/SAM which is all the same in these two nodes.

\section{THE STRUCTURE OF SAM}

Security Account Manager (SAM) is a HIVE file which is consists of HBINs and CELLs. In this section, the data structure of SAM will be analyst.

\section{A. The HIVE Header}

The HIVE file header is the first block of hive which is also named base block. Table I list the content of the HIVE header.

TABLE I: HIVE HEADER

\begin{tabular}{cccl}
\hline \hline Offset & Size & Name & \multicolumn{1}{c}{ Notes } \\
\hline $0 \times 00$ & 4 & regf_id & signature “ regf" \\
$0 \times 04$ & 8 & timestamp & last modify date \\
$0 \times 0 \mathrm{C}$ & 4 & version & HIVE version \\
$0 \times 10$ & 4 & ofs_rootkey & offset of 1st key record \\
$0 \times 14$ & 460 & name & filename and path \\
$0 \times 1 \mathrm{E} 0$ & 4 & checksum & checksum \\
\hline \hline
\end{tabular}

\section{B. $H B I N$}

HBIN is the storage units of HIVE internal data files and always use block (4KB size) as allocation unit. When expanding the HIVE file for the CELLs, the real allocation 
storage space will be extended to the border of next block [3], [4]. Table II shows the content of HBIN.

TABLE II: HBIN CONTENT

\begin{tabular}{cccl}
\hline \multicolumn{3}{c}{ TABLE II: HBIN CONTENT } \\
\hline Offset & Size & Name & \multicolumn{1}{c}{ Notes } \\
\hline $0 \times 00$ & 4 & hbin_id & signature " hbin " \\
$0 \times 04$ & 4 & ofs_self & offset to itself \\
$0 \times 08$ & 4 & ofs_next & relative offset to next HBIN \\
$0 \times 0 \mathrm{C}$ & 4 & firstlink & First data block \\
\hline \hline
\end{tabular}

\section{CELL}

The registry data in the HIVE is organized by CELLs. However the CELL storage space is not certain which are depended on the CELL data and types [3]. Each CELL contains a key, a value, a security description, a subkey-list or a value-list and the corresponding CELL which is named key CELL, value CELL, security description CELL, subkey-list CELL and value-list CELL.

The connection between HBINs and CELLs is shown as Fig. 1.

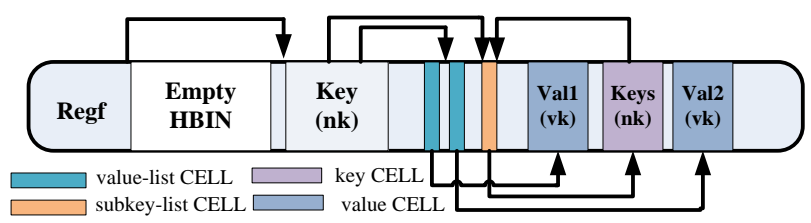

Fig. 1. Connection between HBINs and CELLs.

The above Fig. 1 shows that each registry key record ("nk") can be described as follows:

1) If it has any values, a pointer to a value-list. The value-list in turn points to a number of value records ("vk").

2) If it has any subkeys, a pointer to a subkey-list. The subkey-list in turn points to a number of subkey records which are of course key records ("nk");

3) Each subkey record has a pointer that points back to its parent key record.

\section{WINDOWS ACCOUNT PASSWORD ENCRYPTION}

Microsoft Windows operating system mainly used one-way hash algorithm to encrypt account password, and the LM hash and NTLM hash encryption are the mostly used to integrate single sign-on mechanism in the Microsoft systems [5]. The LM hash is used to encryption logon account in Windows XP, Windows 2000 and Windows 2003. What's the encryption process is:

1) Password converted to upper case;

2) Password is null-padded or truncated to 14 bytes;

3) Password is split into two halves of 7 bytes each;

4) Two DES keys are created, one from each 7 byte half:

- convert each half to a bit stream;

- Insert a zero bit after every 7 bits.

5) Each DES key is used to encrypt a preset ASCII string (KGS!@\#\$\%), resulting in two 8-byte ciphertext values;

6) Concatenate the two 8-byte ciphertext values as the LM hash.

LM stored passwords have a few distinct disadvantages:
1) Passwords are not case sensitive;

2) Password are split into 7 chars and hashed separately, making brute force trivial;

3) Passwords are limited to a maximum of 14 characters in length.

To strengthen the security of the account password, Microsoft add a new encryption method in early systems which named NTLM. NT LAN Manager (NTLM) is a challenge-response protocol that used throughout Microsoft's systems as an integrated single sign-on mechanism. Meanwhile Windows NT-based operating systems up through and including Windows Server ${ }^{\mathrm{TM}} 2003$ store two password hashes for keep compatibility, the LAN Manager (LM) hash and the Windows NT hash. Starting in Windows Vista ${ }^{\mathrm{TM}}$, the capability to store both is there, but LM hash is turned off by default. The NT hash is a straight MD4 hash of the plaintext password. It supports all Unicode characters and passwords up to 256 characters long [5]-[7]. The NTLM response is calculated as follows:

1) The MD4 message-digest algorithm is applied to the Unicode mixed-case password. This results in a 16-byte value;

2) The 16-byte NTLM hash is null-padded to 21 bytes;

3) This value is split into three 7-byte thirds;

4) These values are used to create three DES keys (one from each 7-byte third);

5) Each of these keys is used to DES-encrypt the challenge from the Type 2 message (resulting in three 8-byte cipher text values);

6) These three cipher text values are concatenated to form a 24-byte value. This is the NTLM response.

\section{OBTAIN ACCOUNT INFORMATION AND CLEAR PASSWORD FROM SAM DATABASE}

\section{A. The Content of Project $F$ and $V$}

As has been stated before in this article, Security Account Manager (SAM) is a hierarchical database which is used to store user's account information. The SAM in the registry is organized as a tree and is analogous to a file system as shown in Fig. 2.

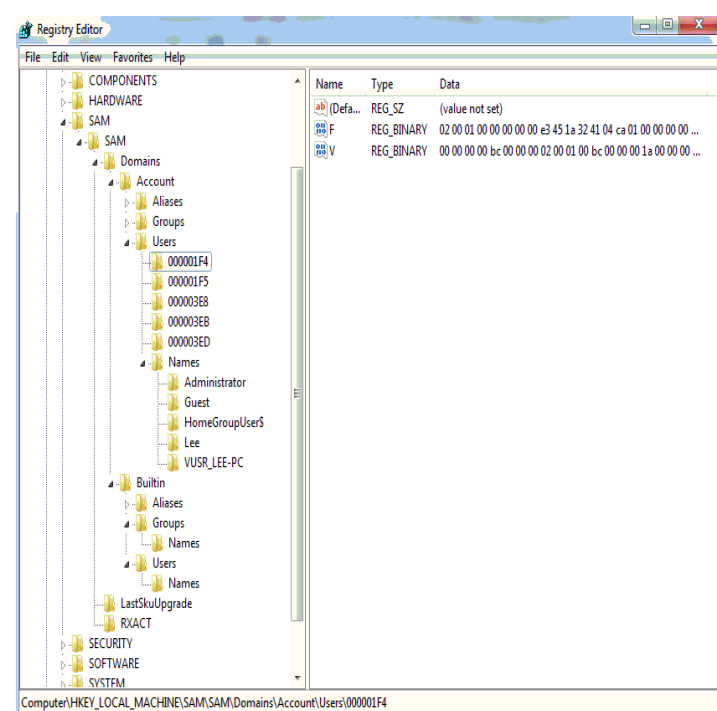

Fig. 2. The hierarchical tree of SAM. 
In the entire SAM database, the main contents of the account are under the node of /Domain/Account/Users. Each account following two sub-items, F and V. Saved in the project $\mathrm{F}$ is the account registry records, such as the last login time, lockout time, the failed login count, total logins since creation and so on. Project V saves the basic information of the account, like user name, full name, contents, group ownership, password hash, etc. Table III and Table IV show the main contents of project $\mathrm{F}$ and $\mathrm{V}$, respectively.

TABLE III: THE PROJECT F CONTENT

\begin{tabular}{cccl}
\hline \multicolumn{4}{c}{ TABLE III: THE PROJECT F CONTENT } \\
\hline \hline Offset & Size & Name & \multicolumn{1}{c}{ Notes } \\
\hline $0 \times 00$ & 8 & t_lockout & time of lockout \\
$0 \times 08$ & 8 & t_creatio & time of account creation \\
$0 \times 10$ & 8 & t_login & time of last login \\
$0 \times 18$ & 4 & rid & RID \\
$0 \times 1 C$ & 2 & ACB_bit & account type and status \\
$0 \times 1 E$ & 2 & failedent & count of failed logins \\
$0 \times 20$ & 2 & logins & total logins since creation \\
\hline \hline
\end{tabular}

TABLE IV: THE PROJECT V CONTENT

\begin{tabular}{cccl}
\hline \hline Offset & Size & Name & \multicolumn{1}{c}{ Notes } \\
\hline $0 \times 00$ & 4 & username_ofs & account offset \\
$0 \times 04$ & 4 & username_len & account length \\
$0 \times 08$ & 4 & fullname_ofs & full username \\
0×0C & 4 & fullname_len & full username length \\
$0 \times 10$ & 4 & comment_ofs & comment \\
$0 \times 14$ & 4 & comment_len & comment length \\
$0 \times 18$ & 4 & homedir_ofs & home directory \\
$0 \times 1 C$ & 4 & homedir_len & home directory length \\
$0 \times 28$ & 4 & lmpw_ofs & LM hash offset \\
$0 \times 2 C$ & 4 & lmpw_len & LM hash length \\
$0 \times 30$ & 4 & ntpw_ofs & NTLM hash offset \\
$0 \times 34$ & 4 & ntpw_len & NTLM hash length \\
\hline \hline
\end{tabular}

\section{B. Obtain Account Information}

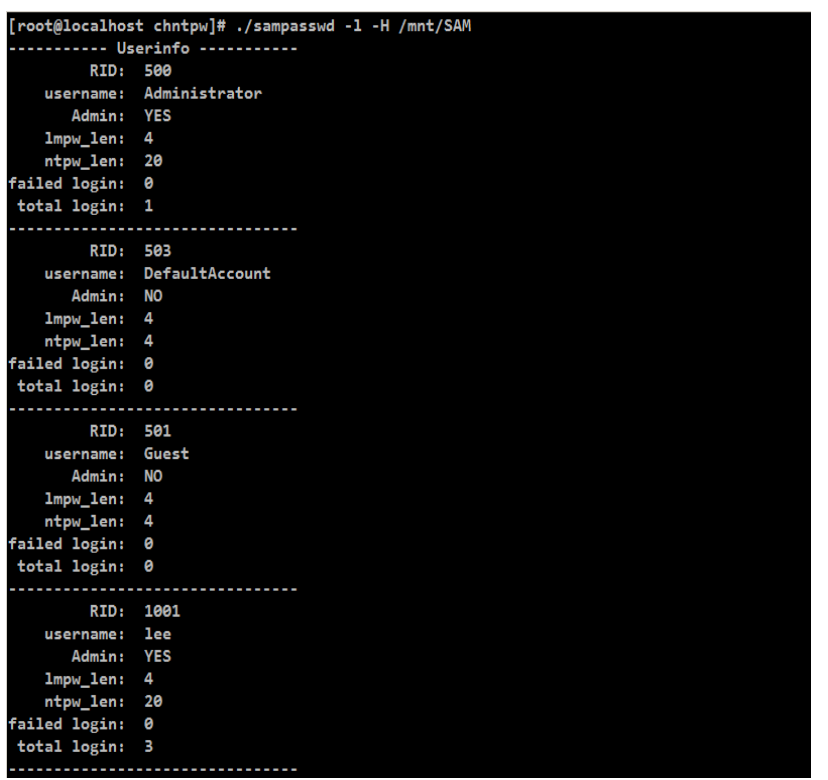

Fig. 3. Obtain information from SAM.
To obtain the account information from SAM database, we first mount E01 disk image file use mount image pro and then mount the SAM database file in the virtual machine. Then, the account information can be obtained from the SAM database shows in Fig. 3.

\section{Cracking Windows Passwords}

There are a few ways to crack windows account password. The mostly used ways are brute-force attacks and dictionary attacks. Brute-force attacks is an application of brute-force search by generate all possible passwords and calculate their hash values and compare this hash value to the hash values that store in the system password database. Dictionary attack is based on trying all the strings in a pre-arranged list, typically derived from a list of words such as in a dictionary. Another effective cracking is known as rainbow tables algorithm which is a list of all possible plaintext permutations of encrypted passwords specific to a given hash algorithm. However, in this paper, we set the length of LM hash and NTLM hash as null that stored in the SAM database to clear the account password so that we can login the windows system without account passwords [1], [8], [9]. The data structure of project $\mathrm{V}$ likes:

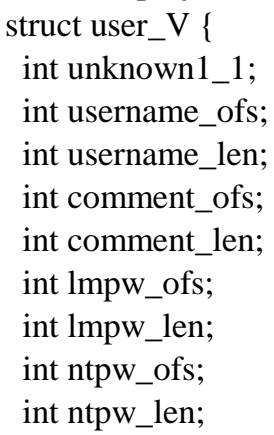

In this data structure, through set lmpw_len=0 and ntpw_len=0 can clear Windows account password like Windows 7, Windows 8 and the latest operating system Windows 10.

\section{CONCLUSION}

In this paper, we first analysis the structure of SAM and discusses the encryption algorithm used in Windows. Then, makes an experiment to show how to obtain the user account information from SAM database and crack the account password. By using the method what I mentioned above, we can crack almost all the Windows account passwords including Windows 7, Windows 8 and Windows 10 which is the latest operating system of Microsoft. However, due to the Windows closure, many data in the SAM database is still unknown what's the meaning it have. And deep research the Windows SAM and obtain more information will be the future work.

\section{REFERENCES}

[1] N. Peter, "The internal structure of the windows registry," Defence College of Management and Technology, Dept. of Informatics and Sensors, Cranfield University, 2009. 
[2] N.-H. Petter. (2008). Offline NT password \& registry editor. [Online]. Available: http://www.pogostick.net/ pnh/ntpasswd/

[3] J. Johansson. (2006). Security watch: The most misunderstood windows security setting of all time. [Online]. Available: https://technet.microsoft.com/en-us/magazine/2006.08.securitywatch. aspx

[4] C. K. Goel and G. Arya, "Hacking of passwords in windows environment," International Journal of Computer Science \& Communication Networks, 2012.

[5] G. Eric. (2006). The NTLM authentication protocol and security support provider. [Online]. Available: http://davenport.sourceforge.net/ntlm.html

[6] T. D. Morgan. (2009). The windows NT registry file format version 0.4 [Online]. Available: http://sentinelchicken.com/data/TheWindowsNTRegistryFileFormat.p df

[7] V. Jos. (1997). On NT password security. Open Solution Proviers. [Online]. Available: http://www.osp.nl/infobase/ntpass.html

[8] Remah. (2014). Deeper into the windows registry. [Online]. Available: http://www.techs-upportalert.com/content/deeper-windows-registry.ht $\mathrm{m}$

[9] B. Randhir, N. Kumar, and S. Sharma, Analysis of Windows Authentication Protocols: NTLM and Kerberos, 2014.

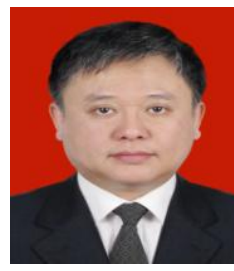

Jiang Du was born in 1969 of Chongqing, China and graduate from Southwest Jiaotong University in 1991. After graduation, he work for Chongqing University of Posts and Telecommunications and server as a lecturer in Telecom Engineering Department. In 1995, he studied for computer master's degree in Inha University and got the master's degree in 1997. In 2010, he was promoted to full professor and major field include information security, computer network and embedded systems.

In 2001, he won the second prize of military science and technology progress. In 2009, he won the first award of Chongqing science and technology progress. In 2010, he obtained the second prize of national science and technology progress.

At present, Prof. Jiang was the senior member of China Computer Institute and the committee member of Computer Security Committee.

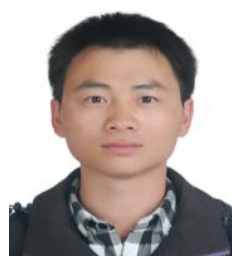

Jiwei Li was born in 1990 of Yunnan province. And now is a master student of Chongqing University of Posts and Telecommunications. The search field is information security. 